\title{
The Solar Wind Ionic Charge States During the Ulysses Pole-to-Pole Pass
}

\author{
Yuan-Kuen Ko ${ }^{1,2}$, George Gloeckler ${ }^{1,3}$, Christina M. S. Cohen ${ }^{4}$, Antoinette B. Galvin ${ }^{5}$ \\ IDepartment of Atmospheric, Oceanic and Space Sciences, University of Michigan, Ann Arbor, MI 48109 \\ ${ }^{2}$ now at Harvard-Smithsonian Center for Astrophysics, Cambridge, MA 02138 \\ ${ }^{3}$ Department of Physics, University of Maryland, College Park, MD 20742 \\ ${ }^{4}$ Space Radiation Laboratory, California Institute of Technology, Pasadena, CA 91125 \\ ${ }^{5}$ Institute for the Study of Earth, Oceans, and Space, University of New Hampshire, Durham, NH 03824
}

\begin{abstract}
We analyze and compare the ionic charge composition data for different types of the solar wind which the Solar Wind Ion Composition Spectrometer on Ulysses observed during the pole-to-pole pass of its primary mission. The implications on the electron temperature, electron density and ion outflow velocity from the corresponding solar wind source regions are also discussed. We find that the electron temperature in the source region of the slow solar wind is higher than that in the coronal hole. We also find a possible north-south asymmetry in the electron temperature that may be correlated to the north-south asymmetry in the solar wind speed found in the SWOOPS/Ulysses data. Based on our data without clear constraints from other coronal observations, it is found that the electron density may be higher, or the heavy ion outflow velocities may be lower toward lower heliographic latitude.
\end{abstract}

\section{INTRODUCTION}

Ulysses was launched in October 1990 and after the Jupiter swingby in February 1992, the spacecraft was deflected out of the ecliptic plane over the south pole of the Sun where a maximum southern latitude of $80^{\circ}$ was reached in September 1994. The pass from the south pole through the equatorial plane over the north pole of the Sun was rapid with Ulysses reaching the highest northern latitude of $80^{\circ}$ in July 1995 . McComas et al. [1998](1) have shown a polar plot of the solar wind proton speed observed by the solar wind plasma instrument (SWOOPS) on Ulysses since launch. It gives a clear structure of the observed solar wind in heliographic latitude during its primary mission. The data from the instruments onboard Ulysses are therefore unique in exploring the properties of the solar wind and the heliospheric structure for a large range of the heliographic latitude. In this paper, we analyze the ionic charge composition data in both the slow and fast solar wind measured by the Solar Wind Ion Composition Spectrometer (SWICS)(2) on Ulysses during its poleto-pole fast scan. During this time, the spacecraft moves from $25^{\circ}$ south to $20^{\circ}$ north around its perihelion (1.34 AU) (Figure 1). We also compare these data with the long-time averaged ionic charge states in the high speed solar wind from the south and north polar coronal holes.
Under steady state condition, the solar wind ionic charge states are generally 'frozen-in' within 5 solar radii of the Sun. The ions then stay in their frozen-in states in the solar wind flow through the interplanetary space. Therefore the observed in-situ ionic charge states are a good probe to the physical properties in the inner solar corona where the ions' freezing-in process occurs. Different ionic charge composition in different types of the solar wind thus implies that the physical properties are different in these solar wind source regions.

\section{DATA SELECTION}

The types of the solar wind and the time periods selected in this study are described below (Fig.1):

[1] Slow solar wind:

Four periods of the slow solar wind are selected for which $\mathrm{v}<400 \mathrm{~km} / \mathrm{s}$. They are then summed up to represent the 'averaged slow wind' denoted as Slow.

[2] Fast solar wind at low latitude:

Two periods of the fast solar wind are selected for which $v>500 \mathrm{~km} / \mathrm{s}$. They are then summed up to represent the averaged fast wind denoted as Fast. During both periods, the measured magnetic polarity is the same as that of the fast wind from the south polar coronal hole. Mapping the solar wind back to the source region on the Sun, various solar magnetic field models have shown that this fast wind comes from near the 


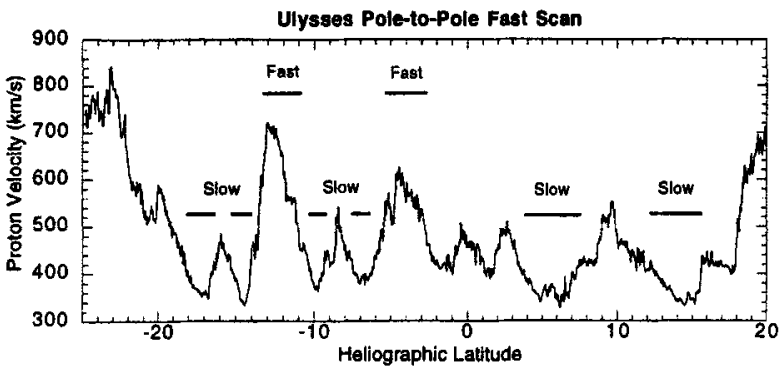

FIGURE 1. The solar wind proton speed measured by SWICS/Ulysses as a function of the in-situ helio graphic latitude during the Ulysses pole-to-pole fast scan in February and March, 1995. The time periods selected for analysis of the slow and fast solar wind are also marked.

boundary or the equatorial extension of the south polar coronal hole (3). On the other hand, Woo and Habbal [1997](4) suggested from the Ulysses radio occultation measurements that this fast wind can originate from much lower latitude on the Sun.

To compare the above charge composition data with those from the polar coronal holes, we also extract the fast solar wind data when Ulysses was at high south and north heliographic latitudes.

[3] Fast solar wind from the south polar coronal hole:

About 388.5 days of raw data are extracted which cover heliographic latitudes from $48^{\circ}$ south to $80^{\circ}$ south and back to $30^{\circ}$ south for which $v>700 \mathrm{~km} / \mathrm{s}$. We denote this data as South.

[4] Fast solar wind from the north polar coronal hole:

About 474 days of raw data are extracted which cover heliographic latitudes from $30^{\circ}$ north to $80^{\circ}$ north and back to $30^{\circ}$ north for which $\mathrm{v}>700 \mathrm{~km} / \mathrm{s}$. We denote this data as North.

Note that isolated periods of high speed solar wind associated with coronal mass ejections (CMEs) were detected during the time when Ulysses was immersed in the fast streams from the south polar coronal hole (5). In our South data, we do not exclude such CMEassociated fast wind data. Since our selected data are overwhelmed by the polar coronal hole related fast wind, the charge composition data analyzed here should not be affected by the few CME-associated events.

\section{DATA ANALYSIS}

The pulse height analysis data (PHA data) were extracted from the Experiment Data Records (EDRs) processed at the University of Maryland. Mass (M) and mass-per-charge $(\mathrm{M} / \mathrm{Q})$ values were determined for each event from these data. For events falling within several 'ion boxes' (as defined by their $M / Q$ and $M$ boundaries), were then converted into ion fluxes by correcting for event priorities, instrument efficiency and duty cycle. For each ion box, we fit the data with a number of 2-D Gaussians each of which corresponds to one particular ion believed to contribute to the flux in this ion box. Each 2-D Gaussian (i.e. ion) is characterized by five parameters: its volume in the $M / Q-M$ space, its $M / Q$, the FWHM (Full-Width-Half-Maximum) of $M / Q$, its mass and the FWHM of mass. The best-fit parameter values are calculated by using a least squares fit technique to fit the sum of the Gaussians to the ion flux matrix in the ion box. The ionic fractions for a given element are then calculated from the volumes of all best-fit 2-D Gaussians corresponding to this element. See Cohen [1995](6) for the full description of this 2-D Gaussian fitting technique and Ko et al. [1999](7) for a more detailed description of this data analysis.

\section{RESULTS AND DISCUSSION}

We compare the charge composition from different types of the solar wind (fast wind from the north and south polar holes and low latitude coronal holes, and slow wind). We start the comparison in the context of the 'freezing-in temperatures'. We will then discuss how the difference in the ionic charge states infers the difference in the physical conditions between respective solar wind source regions. For a more detailed results and analysis, see Ko et al. [1999](7).

The freezing-in temperature of a given observed ionic ratio is defined as the electron temperature at which the abundance ratio of two neighboring charge states in ionization equilibrium is the same as the observed ratio. And the ionic charge composition in ionization equilibrium move toward higher charge states as the electron temperature increases.

Figures 2 compares the $\mathrm{C}, \mathrm{O}, \mathrm{Si}$ and $\mathrm{Fe}$ charge states between South, North, Fast, and Slow. It shows that (we use South data as reference):

[1] The freezing-in temperatures are slightly lower for North than that for South. This is true for all the four elements.

[2] The freezing-in temperatures of $\mathrm{C}$ and $\mathrm{O}$ ions are higher for Fast than those for South, but the freezing-in temperatures of $\mathrm{Si}$ and $\mathrm{Fe}$ ions are lower for Fast than those for South.

[3] In the slow wind, the freezing-in temperatures are obviously higher than in the fast wind for all elements. For example, the $\mathrm{O}^{+7} / \mathrm{O}^{+6}$ ratio corresponds to a freezing-in temperature of $1.75 \times 10^{6} \mathrm{~K}$ for Slow and $1.17 \mathrm{x}$ $10^{6} \mathrm{~K}$ for South. We note that the charge states from the south polar coronal hole can be roughly fitted by one electron temperature (8). This is not the case for $\mathrm{Si}$ and $\mathrm{Fe}$ ions in the slow wind (9).

We have shown that the freezing-in temperatures of $\mathrm{C}$ and $\mathrm{O}$ ions are such that $T_{f}($ North $)<T_{f}($ South $)<$ $T_{f}($ Fast $)<T_{f}($ Slow $)$ and the freezing-in temperatures of $\mathrm{Si}$ and $\mathrm{Fe}$ ions are such that $T_{f}$ (Fast) $<T_{f}$ (North) $<$ $T_{f}($ South $)<T_{f}($ Slow $)$. This difference in the freezing-in temperatures, i.e. the ionic charge composition, directly 

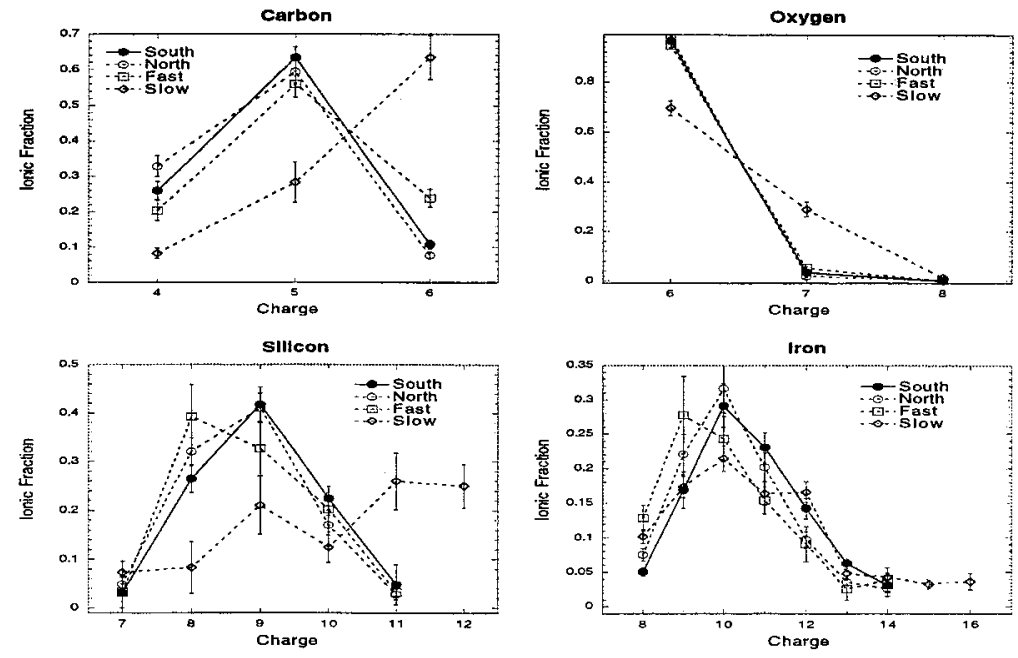

FIGUR 2. The C, O, Si, and Fe charge compositions for the South, North, Fast and Slow data.

reflects the difference in the physical properties, namely the electron temperature, the electron density and the ion outflow velocities in the freezing-in regions of these ions. We stress that the difference in the freezingin temperatures does not necessarily infer the difference in only the electron temperature. Note that possible deviation from Maxwellian for coronal electrons can also affect the observed charge states $(10,11)$. The velocity distribution may also be different in the fast and slow wind. We will not discuss this effect in this work.

In a steady state and radial outflow, the evolution of the solar wind ionic charge states with heliocentric height depends on three physical quantities in the ions' freezing-in region: the electron temperature $T_{e}$, the electron density $n_{e}$ and the ion outflow velocity $u(12)$. We adopt a 'base model' of $\left[T_{e}(r), n_{e}(r), u(r)\right]$ which is among those that best-fit the fast wind charge state data from the south polar coronal hole (13). Note that the electron temperature profile has a local maximum in the freezing-in region. We then calculate the frozen-in ionic charge states when (a) $T_{e}$ is 1.2 times higher, (b) $n_{e}$ is 2 times higher, or (c) $u$ is 2 times higher, while the other two parameters are kept the same. Figure 3 shows the results for $\mathrm{C}$ and $\mathrm{Fe}$ ions. $\mathrm{C}$ and $\mathrm{Fe}$ ions are chosen to illustrate the difference in the change in the charge

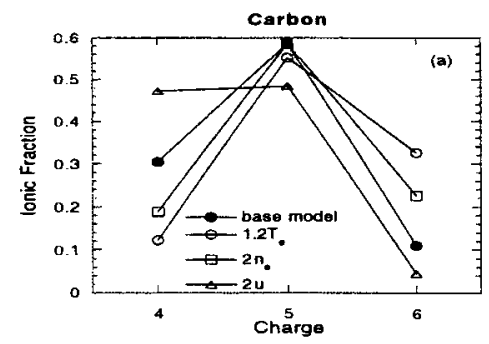

composition when the ions are freezing-in before (C) or after $(\mathrm{Fe})$ the temperature maximum. We can see that (a) when $T_{e}$ is higher, both $\mathrm{C}$ and $\mathrm{Fe}$ charge composition move toward higher charge states, (b) when $n_{e}$ is higher, $\mathrm{C}$ ions move toward higher charge states but $\mathrm{Fe}$ ions move toward lower charge states, and (c) when $u$ is higher, $\mathrm{C}$ ions move toward lower charge states but $\mathrm{Fe}$ ions move toward higher charge states. Note that the effects of higher electron density and lower ion velocity are similar. The above results can be understood by considering the shift in the ions' freezing-in region when these physical quantities are changed. Therefore, to compare the physical conditions in different solar wind source regions where a temperature maximum likely exists, we need the charge composition data of at least two species -- one which freezes-in at one side of the temperature maximum (e.g. C) and the other which freezes-in at the other side of the temperature maximum (e.g. Fe). Based on our data and the above analysis, we discuss in the following the implied difference in the physical properties among these solar wind source regions.

The Slow Wind and the Fast Wind

The electron temperature corresponding to the source

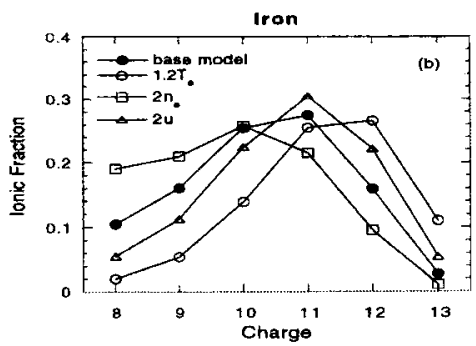

FIGURE 3. The change in the frozen-in ionic charge states of $\mathrm{C}$ and Fe ions compared with the base model $\left[T_{e}(r), n_{e}(r)\right.$ and $u(r)$ ] (filled circles), when $T_{e}(r)$ is 1.2 times (open circles); or $n_{e}(r)$ is two times higher (open squares); or $u(r)$ is two times higher (open triangles), with the other two parameters fixed. 
region of the slow solar wind is higher than that for the fast solar wind from the coronal holes. Note that if the slow solar wind is indeed associated with the streamer region, we expect that the electron density is also higher (and the ion velocity may be slower) than in the coronal hole. Also the temperature profile of the source region of the slow solar wind may not have a temperature maximum. However, since the freezing-in temperatures of the slow wind are all significantly higher for the four elements, the electron temperature in the slow wind source region is certainly hotter than in the coronal hole region.

\section{The North and South Polar Coronal Holes}

The electron temperature in the north polar coronal hole seems to be slightly cooler than the south polar coronal hole during this time. This would suggest that either there is intrinsic difference in the temperature of the two polar coronal holes, or this difference is caused by time variation $(9,14)$. Goldstein et al. [1996](15) have also shown a modest north-south high latitude asymmetry that the proton velocity above 40 degrees in latitude is $13 \mathrm{~km} / \mathrm{s}$ to $24 \mathrm{~km} / \mathrm{s}$ greater at northern latitudes than at southern latitudes. It suggests the possibility that the electron temperature in the inner corona is correlated with the solar wind speed, namely, that higher solar wind speed is related to lower electron temperature in the corona. This may have implications on the coronal heating and solar wind acceleration mechanisms in the inner coronal regions.

\section{Latitudinal variations in the polar coronal hole}

Since the source region for the Fast data is at the lower latitude in the south polar hole, the difference between the Fast and South data implies that there is latitudinal variation in the physical conditions in the polar coronal hole. Galvin and Gloeckler [1998](14) have also found similar heliographic latitudinal variations in the $\mathrm{Si}$ and $\mathrm{Fe}$ ionic charge states in the north polar coronal hole. If we assume the electron temperature does not change along the latitude in the polar coronal hole, this variation in the ionic charge states implies that the electron density is larger or the ion outflow velocity is smaller toward lower latitude. Guhathakurta and Holzer [1994](16) found very little intrinsic latitudinal variation in the electron density within the polar coronal hole. On the other hand, Woo and Habbal [1997](4) found that the electron density increases from the high latitude polar coronal hole toward the low latitude streamer belt region. Strachan et al. [1999](17) have shown from SOHO/UVCS observations the variation in the $\mathrm{O}^{+5}$ outflow velocity as a function of latitude. But it is not clear if there exists systematic latitudinal dependence of the $\mathrm{O}^{+5}$ outflow velocity within the polar coronal hole. Aside from this, there exists no other observation that is capable of deriving outflow velocities of heavy ions. Note that the solar wind proton velocity is smaller in the Fast data than in the South data (cp. Fig. 1). This may indicate that the ion velocity is correspondingly smaller in the source region of the Fast data.

It should be emphasized that the three physical quantities that affect the freezing-in process of the ions (i.e. $T_{e}, n_{e}, u$ ) can all be responsible for the difference in the observed ionic charge states along the heliographic latitude. The effect of one of them may just outweigh another. Therefore there is a clear need of relying on a combined and coordinated data set of the in-situ ionic charge states and remote-sensing coronal observations to unambiguously relate the latitudinal variations of these physical quantities to the latitudinal variations of the ionic charge states in the associated solar wind.

\section{ACKNOWLEDGMENTS}

This work is supported by NASA/JPL contract 955460 and NAG 5-2810.

\section{REFERENCES}

1. McComas, D. J. et al., Geophys. Res. Lett., 25, 1-4 (1998).

2. Gloeckler, G. et al., Astron. Astrophys. Suppl. Ser., 92, 267-289 (1992).

3. Neugebauer, M. et al., J. Geophys. Res., 103, 14587 14599 (1998).

4. Woo, R., and S. R. Habbal, Geophys. Res. Lett., 24, 1159-1162 (1997).

5. Gosling, J. T., Riley, P., McComas, D. J., and Pizzo, V. J., J. Geophys. Res., 103, 1941-1954 (1998).

6. Cohen, C. M. S., Ph.D. Dissertation, University of Maryland (1995).

7. Ko, Y.-K., G. Gloeckler, Cohen, C. M. S., and Galvin, A. B., submitted to J. Geophys. Res. (1999).

8. Geiss, G. et al., Science, 268, 1033-1036 (1995).

9. von Steiger, R., "Latitude Manifestations of the Solar Wind", in Highlights of Astronomy, IAU Proceedings, Kluwer Academic Publishers, in press, 1998.

10. Esser, R., Edgar, R., J., and Brickhouse, N. S., Astrophys. J., 498, 448-457 (1998).

11. Owocki, S. P. and Ko, Y.-K., in Solar Wind 9 (1999).

12. Hundhausen, A. J., Gilbert, H. E., and Bame, S. J., Astrophys. J., 152, L3-L5 (1968).

13. Ko, Y.-K. et al., Sol. Phys., 171, 345-361 (1997).

14. Galvin, A. B., and G. Gloeckler, "Charge State Composition in Coronal Hole and CME Related Solar Wind: Latitudinal Variations Observed by Ulysses and WIND", in Proceedings 31st ESLAB Symposium 'Correlated Phenomena at the Sun in the Heliosphere and in Geospace', 1998, pp.323-326.

15. Goldstein, B. E. et al., Astron. Astrophys., 316, 296303 (1996).

16. Guhathakurta, M., and Holzer, T. E., Astrophys. J., 426, 782-786 (1994).

17. Strachan, L. et al., submitted to Astrophys. J. (1999). 